\title{
Developing Accessible Mobile Phone Applications: The Case of a Contact Manager and Real Time Text Applications
}

\author{
María Fernanda Cabrera-Umpiérrez ${ }^{1}$, Adrián Rodríguez Castro ${ }^{1}$, \\ Jon Azpiroz ${ }^{2}$, Juan Bautista Montalvá Colomer ${ }^{1}$, \\ María Teresa Arredondo ${ }^{1}$, and Javier Cano-Moreno ${ }^{1}$ \\ ${ }^{1}$ Life Supporting Technologies \\ E.T.S.I. Telecomunicacion. UPM \\ Ciudad Universitaria; 28040 Madrid, Spain \\ ${ }^{2}$ Fundación Vodafone España, \\ Parque Empresarial La Moraleja \\ Avda. de Europa, 1; 28108 Madrid, Spain \\ \{fjcano, jmontalva, chiqui, mta, arodriguez\} @lst.tfo.upm.es, \\ jon.azpiroz@vodafone.com
}

\begin{abstract}
Mobile phones are becoming increasingly popular and are already the first access technology to information and communication. However, people with disabilities have to face a lot of barriers when using this kind of technology. This paper presents an Accessible Contact Manager and a Real Time Text application, designed to be used by all users with disabilities. Both applications are focused to improve accessibility of mobile phones.
\end{abstract}

Keywords: Mobile Application, Accessibility, Real Time Text, Contact Manager, Phone Dialer.

\section{Introduction}

In a mobile world where technology development reaches the market every time faster and mobile subscriptions expects to be at the end of 2010, 5.3 billion [1], people with disabilities face different barriers to access and use mobile devices. Although some of the mobile Operating System (OS) developers are making the effort to build more accessible OS by providing accessibility Application Programming Interfaces (APIs), like the Android and IPhone OS, if the application developed does not make use of these Accessibility APIs or follow accessibility guidelines, most of the effort made is useless.

The accessibility barriers faced by people with disabilities in mobile phones are more critical when it comes to essential applications needed for basic communications, such as dialer, contact manager or instant messengering, this last especially for users with hearing or speech disabilities. To fulfill the accessibility requirements of these applications, an Accessible Contact Manager with phone dialer and a Real Time Text application have been developed within the context of the European funded 
Project AEGIS [2] that seeks to determine whether 3rd generation access techniques will provide a more accessible, more exploitable and deeply embeddable approach in mainstream ICT.

\section{Accessible Real Time Text Application}

This application consists of an instant messaging application in real time. This means that each time the user writes a character, it is automatically received by the recipient. Thus, it is not necessary to press an additional button to send what the user is writing. This application is very useful for users with hearing or speech disabilities as it allows a faster communication process.

This kind of communication works as a simple voice call. To establish the connection the Voice over IP (VoIP) protocol is used. The application connects to the internet network using the mobile phone available network e.g. $3 \mathrm{G}$ or WiFi.

The protocol that has been implemented to allow sending and receiving data in real time is the Real Time Protocol, IETF RFC4103(1), using the control of the connection through the open source SIP server Kamailio [3]. A voice connection is needed to register the mobile device in the server and to send invitations to start a conversation. Once the invitation is accepted, the application provides the user with two text boxes, one to write the messages and the other to show what the received messages, like in a standard chat application.

The connection security and reliability is one of the most important features of the RTT application. This has been guaranteed using the redundancy method of Real Time Protocol (RTP). The other protocol that helps to manage the communication is the Real Time Control Protocol [4] which provides the information about the status of the connection. At the same time, before the real time connection is established, a process for authentication and negotiation of capabilities between each device is required. These steps are carried out through the Session Initiation Protocol and Session Description Protocol [5].

The Graphical User Interface (GUI) has been implemented following accessibility guidelines to ensure a high usability of the application. Furthermore, a configuration screen has been implemented, to allow the user to personalize some parameters like the font size, color theme, icon type or icon size. This is shown in figure 1 .

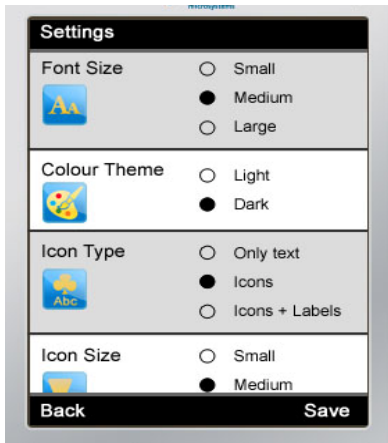

Fig. 1. RTT application: configuration screen 
When all steps to launch the application have been followed (configuration, connection with the server, call and call accepted), the user interface shows the screen divided in two parts, one to write and the other to see what the other user is writing (see figure 2).

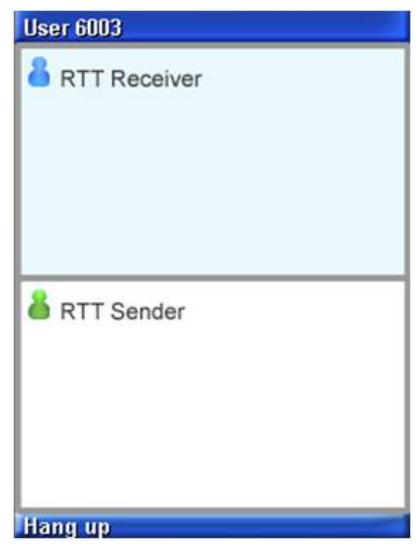

Fig. 2. RTT application: main screen

As it was mentioned before, the message could arrive with some errors, so when the application detects them, it shows a special character to indicate the user that this specific information could be wrong and, if it is possible to solve the error using de redundancy method, the affected characters are replaced by the correct ones. The main innovation of this solution relies on the use of real time text communications in mobile phones.

\section{Accessible Contact Manager Application}

The Accessible Contact Manager with phone dialer has been developed under Android 2.2 OS. The design has followed the process of "Design Thinking" following a path that has as starting point the definition of the problem and the analysis of the state of the art of phone dialer existing in the market. Only the best features of the available applications have been selected, thus discarding those that did not meet accessibility requirements. For example, the attribute that avoids redundancy of buttons functionality has high priority, as well as the location of buttons across the screens. All these characteristics contribute to facilitate the cognitive accessibility of the application.

Furthermore, the icons used are very intuitive always accompanied by explanatory texts which are configurable. This is an accessibility advantage, especially for people with visual and cognitive disabilities. The font size, layout and colors are as well configurable: various types of contacts placements are available on the screen, the location of contacts can be combined with a specific font size, white over black is used or optionally black over white according to the personal preferences and especially based on the user needs (see figure 3 ). 


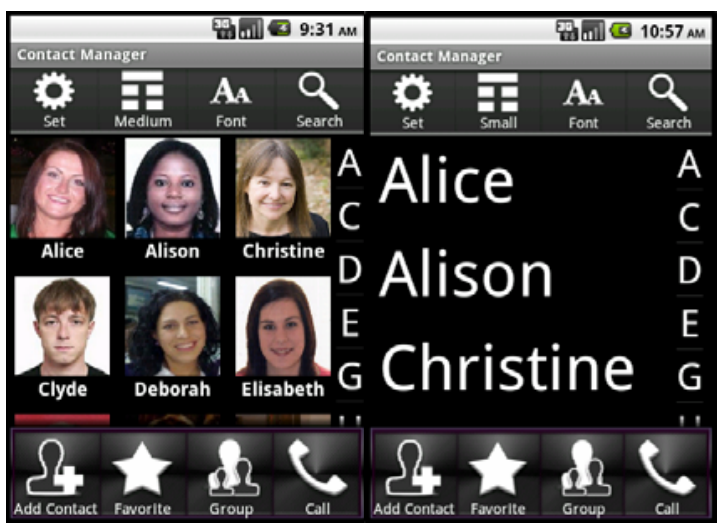

Fig. 3. Contact Manager main screen

All these features implemented are supported by Android guidelines [6], especially buttons on both header and footer, which have the normalized size.

The main functionalities of this application are directly accessed from the main screen, by pressing the buttons of the header and footer. The table below presents the available actions.

Table 1. Main screen available functionalities

\begin{tabular}{lll}
\hline Element & Information & Action \\
\hline $\begin{array}{l}\text { Contacts showed on } \\
\text { the screen }\end{array}$ & $\begin{array}{l}\text { Name and/or photo (depending } \\
\text { on the layout) }\end{array}$ & $\begin{array}{l}\text { OnClick: open the } \\
\text { contact details for } \\
\text { this contact } \\
\text { OnScroll: move the } \\
\text { list to show more } \\
\text { contacts }\end{array}$ \\
\hline Settings & $\ldots$ & $\begin{array}{l}\text { OnClick: open } \\
\text { settings configuration } \\
\text { window }\end{array}$ \\
\hline Layout & Next layout & $\begin{array}{l}\text { OnClick: change to } \\
\text { the next layout } \\
\text { (1x1,2x2,3x3 and } \\
\text { name list without } \\
\text { photo) }\end{array}$ \\
\hline Font & $\ldots$ & $\begin{array}{l}\text { OnClick: change to } \\
\text { the next font size }\end{array}$ \\
\hline Search & $\ldots$ & $\begin{array}{l}\text { OnClick: look for a } \\
\text { contact }\end{array}$ \\
\hline Add Contact & $\ldots$ & $\begin{array}{l}\text { OnClick: open the } \\
\text { add contact window. }\end{array}$ \\
\hline Favourite & $\ldots$ & $\begin{array}{l}\text { OnClick: open the } \\
\text { favorites group } \\
\text { contacts window }\end{array}$ \\
\hline Group & $\ldots$ & $\begin{array}{l}\text { OnClick: open the } \\
\text { group contact } \\
\text { selection window }\end{array}$ \\
\hline
\end{tabular}


Table 1. (continued)

\begin{tabular}{|c|c|c|}
\hline Call & $\ldots$ & $\begin{array}{l}\text { OnClick: call the } \\
\text { selected contact }\end{array}$ \\
\hline Alphabetic list & $\begin{array}{l}\text { Actual position of the contact } \\
\text { list }\end{array}$ & $\begin{array}{l}\text { OnClick: Move the } \\
\text { list of contacts } \\
\text { showing the first } \\
\text { contact which name } \\
\text { starts with the letter } \\
\text { selected. If the action } \\
\text { is a long click, the } \\
\text { font size of the } \\
\text { alphabetic list is } \\
\text { changed } \\
\text { OnScroll: move the } \\
\text { alphabetic list and the } \\
\text { contact list in a } \\
\text { synchronized way }\end{array}$ \\
\hline
\end{tabular}

In order to provide an accessible contact manager, it is extremely important to know which contact is being selected. Therefore, when a new contact is added, the application provides a way to link different features to the same contact. This is achieved associating an audio file to every contact that can be recorded from the contact himself to help people with cognitive or visual disabilities to identify them, or even Text to Speech, audible through the cursor movement or a simple touch over the contacts.

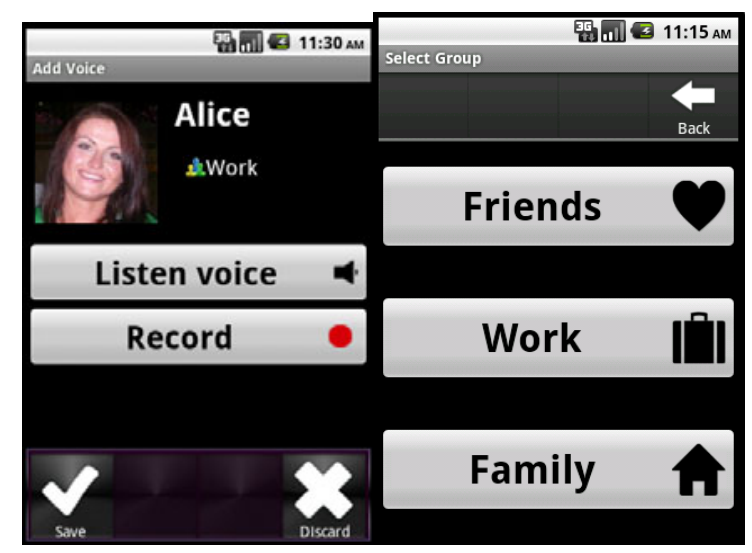

Fig. 4. Listen/Record audio file and Group screens

This feature is very useful especially for blind people or people with cognitive disabilities as it provides an easy way to recognize the contact that is being call. In addition to that, the application allows affiliating each contact to a group (friends, work, family ...), so the user can associate and recognize a contact also through the group in which it is associated (see figure 4). 


\section{Evaluation Results}

Following the release of the first prototypes, different tests have been conducted with a limited number of end users of five category groups: visual, hearing, motor, cognitive and speech/communication disabilities.

According to the preliminary evaluation results, it was agreed that the contact manager has an important number of functionalities that are not included in conventional contact manager applications. Therefore, the response times have not been comparable to these built-in applications but have successfully improved the threshold values. The users found the application easy to navigate and to understand, however it can be hard to use for the users with some hand motor dysfunction.

The deaf and hard of hearing persons showed acceptance of the real time messaging and enjoyed the quickness offered by the application, however some did not like the other person seeing their spelling mistakes.

In general, the Accessible Contact Manager and the Real Time Text applications have proven to be very flexible and adaptable applications for the users.

Within the framework of the AEGIS project, two more evaluation phases will be carried out as well as a final demonstrator that will ensure that the applications are developed according to the preferences of the users.

\section{Conclusions}

This paper has presented two different prototypes of accessible mobile applications. Although the barriers that users have to face when accessing mainstream ICT systems and applications are common in most of the ICTs environments, mobile platforms add some restrictions that should be taken into account when designing accessible applications.

During the evaluation of the first prototypes by the end users, several features that improve the accessibility of the applications have been identified. Even though a great effort has been put to identify accessibility solutions that could improve the user experience, the end-users have identified several modifications to adapt the application to their real needs. The use of a user centred design methodology and the iterative evaluation will guarantee the optimization of the application in future versions

Acknowledgments. This work has been carried out as part of the ÆGIS (Open Accessibility Everywhere: Groundwork, Infrastructure, Standards) project which is partially funded by the European Commission under the Seventh Framework Programme funding for Research on e-Inclusion, grant agreement No. 224348.

\section{References}

1. International Telecomunication Union (2010)

2. AEGIS "Open Accessibility Everywhere: Groundwork, Infrastructure, Standards" project. IST-224348 
3. Mierla, D.G.: Kamailio Project. Techistan Magazine (2009)

4. Hellstrom, G., Jones, E.P.: RFC4103 RTP payload for text conversation (2005)

5. Handley, M., Jacobson, V.: RFC2327 SDP: Session Description Protocol (1998)

6. Inc, Google, User Interface Guidelines. On line at http : / / developer . android. com (last visited January 2011) 